
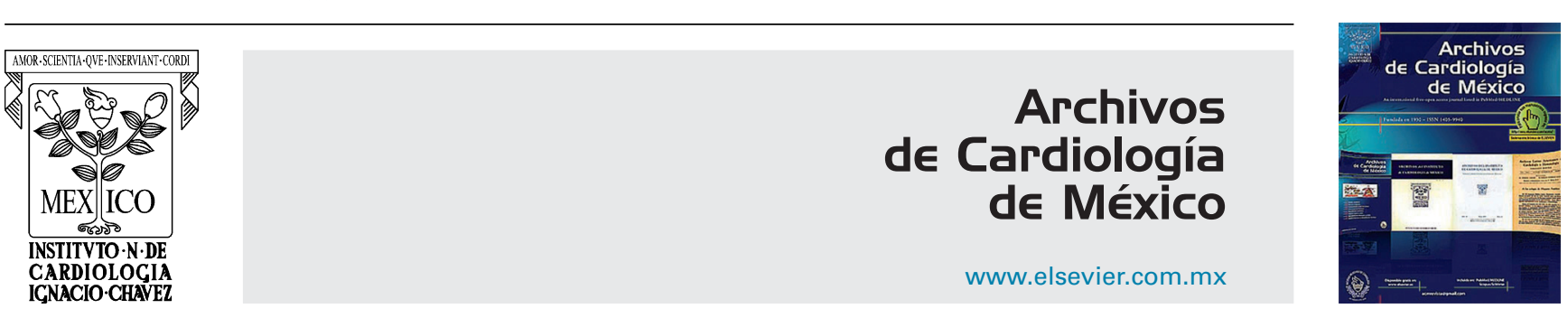

INVESTIGACIÓN CLÍNICA

\title{
Seguridad de la re-esternotomía media en el tratamiento paliativo de pacientes con corazón univentricular
}

\author{
Héctor Díliz-Nava ${ }^{a}$, Isis Meléndez-Sagaón ${ }^{a}$, Orlando Tamaríz-Cruz ${ }^{\mathrm{b}}$, \\ Luis García-Benítez ${ }^{a}$, Aric Araujo-Martínez ${ }^{\mathrm{c}}$ y Alexis Palacios-Macedo ${ }^{\mathrm{a}, *}$ \\ a Cirugía Cardiovascular, Instituto Nacional de Pediatría, Ciudad de México, México

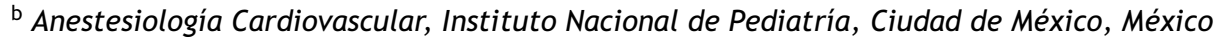 \\ c Terapia Cardiovascular, Instituto Nacional de Pediatría, Ciudad de México, México
}

Recibido el 30 de junio de 2016; aceptado el 4 de octubre de 2016

\author{
PALABRAS CLAVE \\ Re-esternotomía \\ media; \\ Seguridad; \\ Corazón \\ univentricular; \\ Glenn; \\ Fontan; \\ México
}

\begin{abstract}
Resumen
Objetivo: Describir la morbimortalidad operatoria relacionada a re-esternotomía media en pacientes con corazón univentricular en el Instituto Nacional de Pediatría.

Método: Estudio de serie de casos retrospectiva, descriptiva y analítica del año 2001 al 2016. Resultados: Se encontraron 65 pacientes que requirieron 76 procedimientos. Hubo 59 primeras re-esternotomías, con promedio de edad de 36 meses (4-176 meses) y peso $12.2 \mathrm{~kg}$ (3.2-21.5 kg); se realizaron 40 procedimientos de Glenn y 19 procedimientos de Fontan. Se practicaron 17 segundas re-esternotomías, con promedio de edad de 89 meses (48-156 meses) y $22.7 \mathrm{~kg}$ de peso (14.4-41 kg), en quienes se realizaron 17 procedimientos de Fontan. Hubo dos lesiones en primeras re-esternotomías, una lesión a la coronaria derecha con cambios electrocardiográficos, y una apertura incidental de la aurícula derecha que causó hipotensión. Reportamos una muerte secundaria a lesión de la aorta con sangrado masivo durante una segunda re-esternotomía, lo que representa una morbimortalidad del $3.9 \%$.

Conclusiones: Concluimos que la re-esternotomía es un procedimiento seguro en nuestro centro.

(C) 2016 Instituto Nacional de Cardiología Ignacio Chávez. Publicado por Masson Doyma México S.A. Este es un artículo Open Access bajo la licencia CC BY-NC-ND (http://creativecommons. org/licenses/by-nc-nd/4.0/).
\end{abstract}

\footnotetext{
* Autor para correspondencia. División de Cirugía Cardiovascular. Avenida de los Insurgentes Sur 3700-C, Insurgentes Cuicuilco, Delegación Coyoacán, 04530, Ciudad de México.

Correo electrónico: apalaciosmacedo@icloud.com (A. Palacios-Macedo).
} 


\section{KEYWORDS}

Repeat median sternotomy;

Safety;

Univentricular heart;

Glenn;

Fontan;

Mexico
Safety of repeat median sternotomy in the palliative treatment of patients with a univentricular heart

\begin{abstract}
Objective: To establish the morbidity and mortality of patients with univentricular hearts who underwent a repeat median sternotomy at the Instituto Nacional de Pediatría.

Method: A retrospective review was performed on the clinical charts of all patients who underwent a repeat median sternotomy from 2001 to 2016.

Results: Sixty-five patients underwent 76 surgeries by repeat median sternotomy. Fifty-nine patients had a first repeat median sternotomy, with a mean age of 36 months (range: 4-176 months) and a mean weight of $12.2 \mathrm{~kg}$ (range: $3.2-21.5 \mathrm{~kg}$ ). Forty patients had a Glenn procedure, and 19 patients had a Fontan procedure. There were 17 patients with a second repeat median sternotomy, with a mean age of 89 months (range 48-156 months), and a mean weight of $22.7 \mathrm{~kg}(14.4-41 \mathrm{~kg})$. A Fontan procedure was performed on all these 17 patients. A section of the right coronary artery with electrocardiographic changes and a right atrium tear that caused hypotension occurred during first repeat sternotomy. An aortic tear occurred during a second repeat sternotomy with massive bleeding and subsequent death. This represents $3.9 \%$ of re-entry injuries.

Conclusion: It is concluded that repeat median sternotomy is a safe procedure.

( 2016 Instituto Nacional de Cardiología Ignacio Chávez. Published by Masson Doyma México S.A. This is an open access article under the CC BY-NC-ND license (http://creativecommons. org/licenses/by-nc-nd/4.0/).
\end{abstract}

\section{Introducción}

Aproximadamente el $10 \%$ de los defectos congénitos cardiacos pertenecen al grupo de corazones con fisiología univentricular (CFU). Hoy en día se ofrece a estos pacientes un tratamiento paliativo que permite una adaptación progresiva del corazón y los pulmones a una circulación en serie ${ }^{1,2}$. De acuerdo a la base de datos de la Sociedad de Cirujanos Torácicos de Norteamérica (Society of Thoracic Surgeons [STS]), en Estados Unidos y Canadá más del 90\% de los cirujanos prefieren el abordaje por esternotomía media (EM) para el primer paso de la paliación, lo que expone al paciente a re-esternotomía media (RM) en la subsecuente intervención ${ }^{3-5}$. La re-operación en cirugía cardiaca representa un reto técnico que puede asociarse con morbimortalidad perioperatoria significativa. La información respecto a las RM en edad pediátrica es relativamente limitada $^{6,7}$, pero desde los reportes iniciales en 1986 hasta el último trabajo publicado por Jacobs et al. en 2014, en donde se agrupa la experiencia de la STS, se concluye que la RM en pacientes en edad pediátrica es generalmente un procedimiento seguro ${ }^{8-10}$. En México, aunque no existe una base de datos que pueda afirmarlo, la EM parece ser poco utilizada como abordaje quirúrgico en el primer procedimiento paliativo del CFU; esto probablemente por los riesgos asociados a re-intervenciones subsecuentes. Aun así, en el Instituto Nacional de Pediatría hemos adoptado, desde el año 2001, la EM como abordaje inicial por las ventajas que puede ofrecer en el manejo secuencial de estos pacientes.

El presente estudio tiene como objetivo demostrar la seguridad de la RM como abordaje en pacientes con CFU, un grupo que, por las características de su tratamiento paliativo, está expuesto a intervenciones repetidas.

\section{Métodos}

Se realizó de manera retrospectiva un estudio de serie de casos en donde se describe la morbimortalidad operatoria relacionada a RM en pacientes pediátricos con CFU. Se incluyeron los pacientes operados por RM en el Instituto Nacional de Pediatría entre los años 2001 al 2016. Se incluyeron todos aquellos pacientes en edad pediátrica operados por el mismo cirujano mediante RM en cualquier etapa del tratamiento paliativo. Se excluyeron aquellos pacientes cuya re-operación se realizó dentro de los 30 días posteriores a la EM previa. También se describen las variables relacionadas al procedimiento quirúrgico (diagnóstico, tipo de procedimiento, número de RM e intervalo entre las esternotomías) y las complicaciones dentro del quirófano, así como el resultado de la RM practicada.

Se definió morbilidad quirúrgica relevante como aquella que hizo necesaria la canulación de urgencia o que condicionó un estado de choque hipovolémico agudo con necesidad de transfusión aguda de productos hemáticos.

La mortalidad quirúrgica se definió como la relacionada directamente a la RM.

\section{Análisis de resultados}

Se hizo un análisis descriptivo de las variables de interés. Para las variables cualitativas (lesiones a estructuras cardiacas o vasculares, canulación de urgencia, muerte, sexo, tipo de procedimiento, diagnóstico y número de RM) se reportan las frecuencias y las proporciones. Para las variables cuantitativas (edad, peso e intervalo de RM) se reportan las medias y desviaciones estándar. 


\section{Resultados}

Se encontraron 65 pacientes con CFU, los cuales requirieron 76 re-intervenciones por RM: $31 / 65$ (47.6\%) fueron hombres y $34 / 65$ (52.3\%), mujeres. La edad promedio en la primera RM fue de 36 meses $D E \pm 42$ (rango 4-176 meses), y para la segunda RM fue de 89 meses $D E \pm 39$ (rango 48-156 meses). Los diagnósticos más frecuentes de los 65 pacientes fueron atresia tricuspídea, doble entrada de ventrículo izquierdo y atresia pulmonar con septum íntegro (tabla 1).

En relación al motivo de la primera EM, a 22 pacientes se les realizó una fístula sistémico-pulmonar tipo BlalockTaussig modificada, en 16 pacientes bandaje de la arteria pulmonar, a 14 se les realizó el procedimientos de Glenn, se realizaron 4 avances del arco aórtico con bandaje de la arteria pulmonar, y procedimiento de Norwood en 3 pacientes.

La RM se realiza siguiendo varios pasos bien establecidos. El proceso inicia con un estudio de imagen, ya sea angiotomografía o cateterismo cardiaco, para valorar la anatomía y la distancia entre el esternón y las estructuras cardiacas. Antes de iniciar el procedimiento se colocan parches de desfibrilación externos y se tienen en sala cánulas e injertos adecuados para canulación femoral. En nuestra población la alterativa para canulación femoral es a través de un injerto de PTFE suturado de manera termino-lateral, variando el tamaño de acuerdo a la cánula y los flujos requeridos. En ningún caso se anticipa la colocación de cánulas femorales previo a la re-operación. Se incide la cicatriz previa en la piel y se diseca con electrocauterio hasta llegar al esternón. Se retiran los puntos esternales de la cirugía previa. La división del esternón inicia en la parte más inferior de la herida, y se procede a disecar planos laterales por debajo del apéndice xifoides. Una vez liberado este espacio, con cautela se levantan, utilizando retractores, ambos bordes costales, y se extiende la disección lo más lateralmente posible, de preferencia entrando a los espacios pleurales. Utilizando la sierra de repetición se incide la tabla anterior del esternón, y la tabla posterior se corta con tijera Mayo avanzando por segmentos hacia la región superior de la incisión. Durante este proceso el anestesiólogo mantiene presiones positivas al final de la expiración (PEEP) entre 6 y 8 , lo que facilita encontrar un mejor plano de disección. Conforme se abre el esternón, se realiza también disección cefálica y lateral de los tejidos por debajo del esternón, evitando desgarro de la masa ventricular, para paulatinamente ampliar el campo quirúrgico. Una vez dividido por completo el esternón, se terminan de cortar adherencias laterales para poder colocar el separador esternal y se continúa la disección hasta separar completamente la cara anterior del corazón, la aorta y otras estructuras relevantes para realizar el procedimiento.

El intervalo de tiempo promedio entre la EM inicial y la primera RM fue de 31 meses $D E \pm 33$ (rango 3-172 meses). El tiempo promedio entre la primera RM y la segunda RM fue de 64 meses $D E \pm 33$ (rango 35 a 116 meses).

El promedio de peso de los pacientes en la primera RM fue de $12.2 \mathrm{~kg} \mathrm{DE} \pm 9.2$ (rango 3.2 a $41.5 \mathrm{~kg}$ ), mientras que para la segunda RM el peso promedio fue de $22.7 \mathrm{~kg} \mathrm{DE} \pm 8.9$ (rango 14.4 a $41 \mathrm{~kg}$ ).

Respecto al número de RM, 59 (77.6\%) de los abordajes fueron primera RM, de los cuales 40 fueron procedimientos de Glenn y en 19 pacientes se completó la circulación de Fontan. Diecisiete (22.3\%) fueron segundas RM, siendo todas para procedimientos de Fontan.

Se presentó morbilidad relevante en 2 pacientes $(2.6 \%$ del total del grupo estudiado) y muerte relacionada al procedimiento de re esternotomía en otro paciente $(1.3 \%)$.

\section{Morbilidad quirúrgica relevante}

Caso 1. Paciente femenino de 8 años de edad con diagnóstico de canal auriculoventricular desbalanceado con estenosis pulmonar. Fue operada a los 5 años de una fístula

Tabla 1 Datos generales

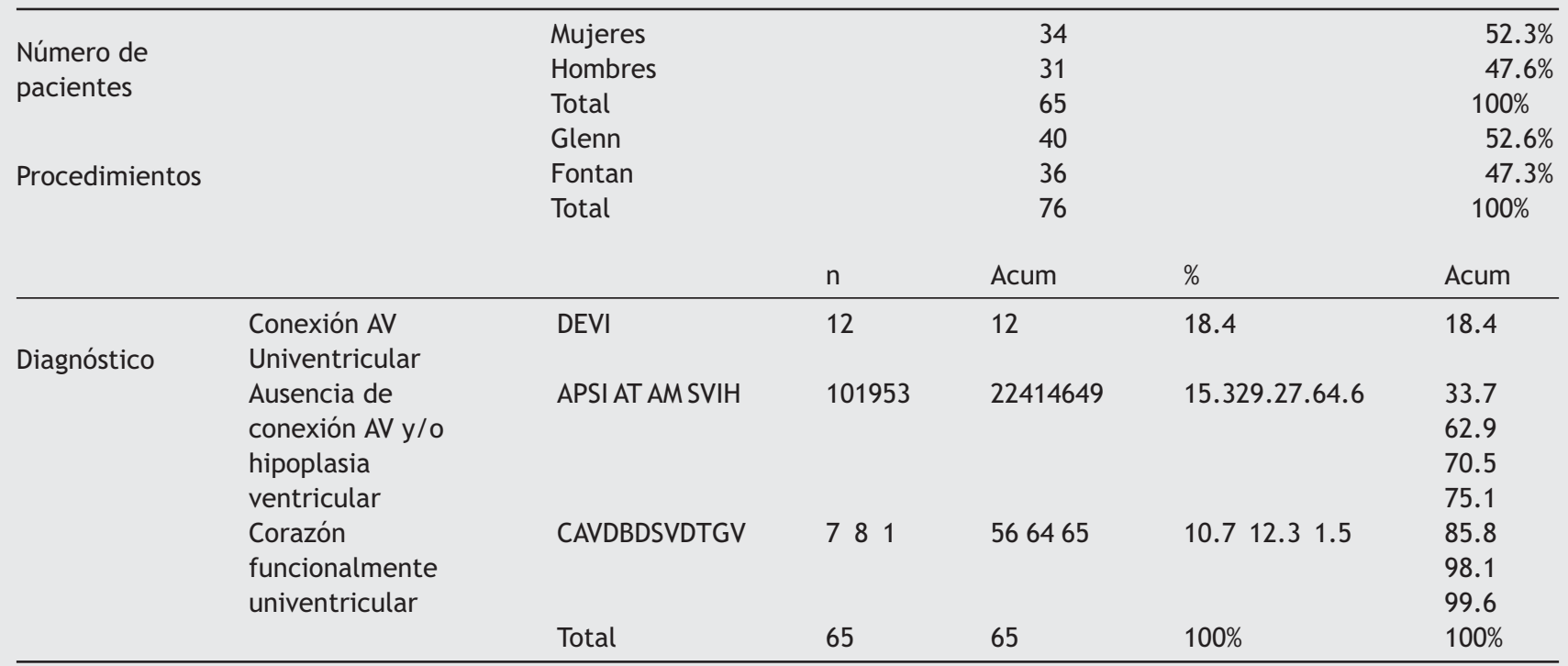

DEVI: Doble entrada ventrículo izquierdo; APSI: Atresia pulmonar con septum íntegro; AT: Atresia tricuspidea; TGV: Transposición de grandes vasos; AM: Atresia mitral; SVIH: Síndrome de ventrículo izquierdo hipoplásico; CAVDB: Canal atrioventricular desbalanceado; DSVD: Doble salida de ventrículo derecho. 
sistémico-pulmonar modificada para promover el crecimiento de la rama pulmonar izquierda. Se realiza primera RM para completar la circulación de Fontan 22 meses posteriores a la cirugía inicial. Durante la disección para separar el corazón del esternón se lesionó la arteria coronaria derecha en su origen. La paciente presentó alteraciones isquémicas cardiacas, por lo que se realizó canulación de urgencia y revascularización miocárdica con colocación de un puente coronario de mamaria derecha. La cirugía transcurrió sin más complicaciones.

Caso 2. Paciente femenino de 5 años con diagnóstico de atresia pulmonar con septum íntegro, en quien al nacimiento se realiza fístula sistémico-pulmonar por toracotomía y posteriormente, mediante EM, procedimiento de Glenn bidireccional al año de edad. Durante la RM para realizar el procedimiento de Fontan se abre la aurícula derecha antes de completar la apertura esternal. A pesar de controlar el sangrado, este causó hipotensión que requirió de transfusión de paquete globular para su corrección. Se completa la cirugía sin otras complicaciones. La orejuela de la aurícula derecha se encontraba muy adherida al esternón debido a un punto de seda colocado en la cirugía previa.

\section{Mortalidad}

Caso 3. Paciente masculino de 5 años de edad con diagnóstico de atresia tricuspídea y estenosis pulmonar. Al nacimiento se realiza una fístula sistémico-pulmonar modificada por EM. A los 13 meses se realiza procedimiento de Glenn sin complicaciones. Cuarenta y tres meses después se planea completar la circulación de Fontan. Al disecar la aorta del esternón se lesiona la aorta, provocando un sangrado masivo que no puede controlarse. Anatómicamente la aorta estaba anterior, muy adherida al esternón.

\section{Discusión}

Los pacientes que nacen con CFU requieren, habitualmente, de varias intervenciones quirúrgicas que permitan establecer una circulación en serie. El éxito depende, en parte, de un buen diseño y planeación de los procedimientos que, si se realizan en tiempo adecuado, pueden contribuir a disminuir la morbimortalidad asociada a la fisiología univentricular.

En México, en algunos centros quirúrgicos se percibe a la RM como un riesgo operatorio significativo y se utiliza con poca frecuencia la EM en la primera etapa de la paliación en pacientes con CFU, aunque a día de hoy no existe un registro de la frecuencia ni del tipo de complicaciones con este abordaje en nuestro medio. En la División de Cirugía Cardiovascular del Instituto Nacional de Pediatría, desde el año 2001 se ha utilizado la EM como procedimiento inicial en este tipo de pacientes, ya que este abordaje permite: 1) el estudio detallado de la anatomía cardiaca; 2) planear el sitio más adecuado para llevar a cabo el procedimiento inicial, así como los subsecuentes; 3) evitar la compresión pulmonar y sus efectos sobre la oxigenación en pacientes con importante desaturación preoperatoria; 4) utilizar circulación extracorpórea en caso de inestabilidad del paciente; 5) ligar el conducto arterioso cuando se requiera ${ }^{4}$, y 6 ) en casos de bandaje de la arteria pulmonar, ajustar con precisión el bandaje, en base a la medición directa de la presión pulmonar y de las saturaciones de oxígeno.

Una de las ventajas de realizar la paliación inicial por toracotomía derecha, en casos de fístula de Blalock-Taussig modificada, es el hecho de poder realizar la anastomosis del injerto a nivel de la arteria subclavia. Esto permite regular mejor el flujo pulmonar, puesto que el calibre limitante es el de la arteria subclavia, lo que evita la posible sobrecirculación pulmonar en neonatos, particularmente los de bajo peso ${ }^{5}$. Aunque esta ventaja es innegable, el abordaje por EM permite habitualmente, con una disección más extendida, realizar también la anastomosis distal del injerto a nivel de la arteria subclavia y no del tronco braquiocefálico en caso de considerarse necesario.

La RM es un procedimiento que sin duda demanda habilidad técnica y una planeación meticulosa con el fin de evitar posibles eventos catastróficos. Sin embargo, los reportes de la literatura concluyen que, llevada a cabo en forma adecuada, presenta una incidencia muy baja de complicaciones directamente relacionadas a este abordaje.

En 1986, DeLeon et al. ${ }^{9}$ reportaron lesión al corazón o grandes vasos en el $5 \%$ de 164 de pacientes sometidos a RM, 16 de ellos con CFU, sin muerte alguna. Más recientemente, Morales et al. ${ }^{5}$ en 2008 y Kirshbom et al. ${ }^{10}$ en 2009 coinciden en una disminución en la incidencia de lesiones de reentrada, con cifras menores al $2 \%$, también sin mortalidad reportada. Jacobs et al. ${ }^{6}$, al analizar la experiencia global reportada en la base de datos del STS, concluyen, de igual forma, que la RM es un procedimiento seguro, y que solo en pacientes que requieren de 5 o más RM el riesgo de complicaciones quirúrgicas se incrementa significativamente.

Por su posición anatómica, la masa ventricular es probablemente la estructura cardiaca más susceptible de lesión durante una RM, la cual ocurre de acuerdo con diversos reportes de literatura con una frecuencia de entre 0.3 y $9.2 \%^{5,7-10}$. En nuestra serie no se presentaron lesiones significativas a esta estructura. Sin embargo, en CFU no es infrecuente que la aorta ascendente sea la estructura más anterior, situación anatómica que la pone en riesgo de ser lesionada durante las subsecuentes RM. En nuestra serie existió una lesión de la coronaria derecha, en su nacimiento de la aorta, que pudo ser resuelta sin más complicaciones con una revascularización de urgencia, y una lesión grave directamente en la aorta que condicionó la única muerte en nuestra serie. A partir de este evento, se afinaron detalles técnicos en nuestra institución, como lo es el cierre del pericardio sobre la aorta cuando el caso lo permita, como una alternativa de bajo costo en lugar de la membrana de PTFE.

En nuestro país, en los últimos años y con la progresiva adquisición de experiencia en todas las áreas relacionadas a la cirugía cardiaca pediátrica, se ha logrado llevar a la paliación definitiva con el procedimiento de Fontan a un número cada vez mayor de pacientes, por lo que es necesario un análisis de los posibles abordajes con sus ventajas y desventajas. En nuestro hospital, durante los primeros años de nuestra experiencia, iniciada en el año 2001, el número de pacientes que lograban llegar al procedimiento de Fontan era relativamente bajo, ya que muchos fallecían entre el primer tiempo quirúrgico y el Glenn, y otros entre este último y el Fontan. Sin embargo, en años recientes un mayor número de pacientes ha podido pasar por las 3 etapas quirúrgicas 
de la paliación. De las 76 RM, el 52.5\% (40 procedimientos) se llevaron a cabo en los últimos 5 años. Al incrementar la sobrevida operatoria de los pacientes con CFU se hace evidente la necesidad de realizar más procedimientos por $\mathrm{RM}^{6}$. En la serie de DeLeon et al. ${ }^{9}$, en 1986, de un total de 164 RM, 16 procedimientos correspondían a pacientes con CFU. En 2009, Kirshbom et al. ${ }^{10}$ reportan 1.000 RM, de las cuales 452 se realizaron en pacientes con las mismas características. Esto hace notar que la RM es un procedimiento que se realiza cada vez con mayor frecuencia, y actualmente representa el abordaje utilizado en aproximadamente el 25\% de todos los procedimientos de cirugía cardiaca en pediatría ${ }^{7,8}$.

\section{Conclusiones}

En pacientes con CFU, la EM para bandaje de arteria pulmonar o realización de fístula de Blalock-Taussig en etapa neonatal ofrece ciertas ventajas sobre la toracotomía. Sin embargo, dicho abordaje se asocia a riesgo de lesiones a estructuras cardiacas o vasculares en RM subsecuentes, que si bien se presentan con una incidencia baja, no son despreciables. Una planeación adecuada de la RM debe permitir llevar a cabo estos procedimientos con una baja probabilidad de complicaciones significativas y de esta forma aprovechar las ventajas que ofrece la EM.

\section{Responsabilidades éticas}

Protección de personas y animales. Los autores declaran que para esta investigación no se han realizado experimentos en seres humanos ni en animales.

Confidencialidad de los datos. Los autores declaran que en este artículo no aparecen datos de pacientes.

Derecho a la privacidad y consentimiento informado. Los autores declaran que en este artículo no aparecen datos de pacientes.

\section{Financiamiento}

Ninguno.

\section{Conflicto de intereses}

Los autores declaramos no tener conflicto de intereses.

\section{Bibliografía}

1. Kaulitz R, Hofbeck M. Current treatment and prognosis in children with functionally univentricular hearts. Arch Dis Child. 2005;90:757-62.

2. Nayak S, Booker PD. The Fontan circulation. Contin Educ Anaesth Crit Care Pain. 2008;8:26-30.

3. Mondésert B, Marcotte F, Mongeon F-P, et al. Fontan circulation: Success or failure? Can J Cardiol. 2013;29:811-20.

4. McKenzie E, Khan M, Samayoa A, et al. The Blalock-Taussig shunt revisited: A contemporary experience. J Am Coll Surg. 2013;216:699-704, discussion 704-6.

5. Morales D, Zafar F, Arrington K, et al. Repeat sternotomy in congenital heart surgery: No longer a risk factor. Ann Thorac Surg. 2008;86:897-902.

6. Jacobs J, Mavroudis C, Quintessenza J, et al. Reoperations for pediatric and congenital heart disease: An analysis of the Society of Thoracic Surgeons (STS) Congenital Heart Surgery Database. Semin Thorac Cardiovasc Surg. 2014;17:2-8.

7. Russell J, LeBlanc J, Sett S, et al. Risks of repeat sternotomy in pediatric cardiac operations. Ann Thorac Surg. 1998;66: 1575-8.

8. Elahi M, Kirke R, Lee D, et al. The complications of repeat median sternotomy in paediatrics: Six-months follow-up of consecutive cases. Interact Cardiovasc Thorac Surg. 2005;4: 356-9.

9. DeLeon SY, LoCicero J III, Ilbawi MN, et al. Repeat median sternotomy in pediatrics: Experience in 164 consecutive cases. Ann Thorac Surg. 1986;41:184-8.

10. Kirshbom PM, Myung RJ, Simsic JM, et al. One thousand repeat sternotomies for congenital cardiac surgery: Risk factors for reentry injury. Ann Thorac Surg. 2009;88:158-66. 